\title{
Electron capture across a nuclear resonance in the strong potential Born approximation $\dagger$
}

\author{
D H Jakubassa-Amundsen $\ddagger$ and P A Amundsen $\S$ \\ ‡ Physik-Department, Technische Universität München, 8046 Garching, Germany \\ $\S$ NORDITA, Blegdamsvej 17, 21 Copenhagen $\varnothing$, Denmark
}

Received 16 May 1984, in final form 20 September 1984

\begin{abstract}
The strong potential Born theory for charge transfer in fast, asymmetric ion-atom collisions has been extended to allow for nuclear resonant scattering using distorted nuclear waves. In the absence of a nuclear resonance, the semiclassical result is recovered. A large variation in the capture probability is found when the projectile energy passes through the resonance. As examples, we present results for the capture from the target $\mathrm{K}$ shell in the collisions of protons with ${ }^{22} \mathrm{Ne}$ and ${ }^{28} \mathrm{Si}$, as well as for capture from the $\mathrm{K}$ and $\mathrm{L}$ shells of ${ }^{58} \mathrm{Ni}$ in collisions with protons, and of ${ }^{16} \mathrm{O},{ }^{20} \mathrm{Ne}$ and ${ }^{28} \mathrm{Si}$ by $\mathrm{He}^{2+}$ impact.
\end{abstract}

\section{Introduction}

Over the last few years the old problem of finding a good perturbation theory for electronic charge transfer in atomic collisions, corresponding to the Born approximation for excitation and ionisation, has finally found an acceptable solution with the emergence of the strong potential Born approximation (SPB, Macek and Shakeshaft 1980, Jakubassa-Amundsen and Amundsen 1980, 1981, Macek and Taulbjerg 1981, Macek and Alston 1982, Jakubassa-Amundsen 1984). The SPB, or various approximations to it, reproduces experimental results very well for $\mathrm{K}$ capture in asymmetric ion-atom collisions both for total cross sections and for the impact parameter dependence (Horsdal Pedersen et al 1982a, b, 1983), and we have recently shown that the same is true for capture at large projectile scattering angles, using a semiclassical sudden approximation to the SPB (Amundsen and Jakubassa-Amundsen 1984a).

During roughly the same period rapid development has also taken place in the new field of measuring interference effects between atomic ionisation and nuclear scattering (Blair et al 1978, Chemin et al 1981, 1982, 1983, Meyerhof et al 1982), and a good theoretical basis for interpreting these experiments has been laid (Blair and Anholt 1982, Feagin and Kocbach 1981, McVoy and Weidenmüller 1982). These kinds of experiments are interesting because they probe the time development of the atomic scattering amplitude during the collision process, and also because of the information they can give on nuclear resonances, some of which are not readily accessible by other methods (Anholt et al 1982). For the same reason, the effect of nuclear resonant scattering on the capture process is an interesting subject too, and we have recently pointed out that the SPB predicts much larger interference effects for capture than those which have been measured for ionisation (Amundsen and Jakubassa-Amundsen 1984b).

$\div$ Supported by the GSI Darmstadt. 
An important observation, originally due to Blair (cf Blair et al 1978), is that although the interference effects under consideration have a perfectly natural and valid classical interpretation as arising from a time delay in the nuclear scattering process (cf Ciocchetti and Molinari 1965), the actual experimental situation is more adequately treated by a quantum mechanical description of the scattering process. Since the observation of time-delay effects will only be possible at large scattering angles, the quantal plane-wave SPB theory (Macek and Shakeshaft 1980, Macek and Alston 1982) has to be extended to include distorted internuclear wavefunctions. However, as the region where the nuclear scattering takes place is small on an atomic scale, knowledge of the asymptotic nuclear functions is sufficient. This leads to a considerable simplification of the calculations.

In applying the theory of electron capture to large angle scattering with a nuclear time delay, there are two extra complications in addition to the use of distorted internuclear waves. One is the proper inclusion of recoil, the effect of which on an electron bound to the projectile (projectile recoil) is so large that a perturbative treatment is invalid (Kocbach and Briggs 1984, Amundsen and Jakubassa-Amundsen 1984a), and which will also cause target excitations (target recoil) which can be handled perturbatively. The other problem is the inclusion of the so-called sticking term (Blair and Anholt 1982; Feagin and Kocbach 1981 call this term the 'nuclear volume term'), which describes the contribution to the ionisation amplitude from the period when projectile and target form a compound nucleus. A proper derivation of the SPB capture amplitude including these two effects by direct analogy with the semiclassical treatment, generalising the Macek-Alston formulation, turns out to be rather involved. Instead, we start with the Faddeev (1961) equations for the full three-body problem, in which case all the pertinent terms emerge in an orderly fashion.

In this paper we give a detailed derivation and extended discussion of the results reported previously (Amundsen and Jakubassa-Amundsen 1984b). In $\S 2$ the quantal version of the SPB theory is formulated, and in $\S 3$ the transfer amplitude is evaluated in the case of capture from the target $\mathrm{K}$ shell. We thereby treat the simplest case of an isolated nuclear resonance $(\S 4)$. In $\S 5$ we present a discussion of our numerical results obtained for $\mathrm{K}$ - and $\mathrm{L}$-shell capture from $\mathrm{O}, \mathrm{Ne}, \mathrm{Si}$ and $\mathrm{Ni}$ by protons and alpha particles. A short conclusion follows $(\$ 6)$. Atomic units $(\hbar=m=e=1)$ are used unless otherwise indicated.

\section{Quantum mechanical description of SPB}

We shall consider the capture of an electron of a heavy target atom (charge $Z_{\mathrm{T}}$ ) by a light projectile (charge $Z_{\mathrm{P}}$ ). In the independent electron model the Hamiltonian for the collision system is

$$
H=T_{\mathrm{N}}+V_{\mathrm{N}}(\boldsymbol{R})+T_{\mathrm{e}}+V_{\mathrm{T}}\left(\boldsymbol{r}_{\mathrm{T}}\right)+V_{\mathrm{P}}\left(\boldsymbol{r}_{\mathrm{P}}\right)
$$

where $H_{\mathrm{N}} \equiv T_{\mathrm{N}}+V_{\mathrm{N}}$ describes the internuclear motion, while $T_{\mathrm{e}}, V_{\mathrm{T}}$ and $V_{\mathrm{P}}$ are the kinetic energy and potential in the target and projectile field, respectively, of the electron under consideration. The coordinates $\boldsymbol{R}, \boldsymbol{r}_{\mathrm{T}}$ and $\boldsymbol{r}_{\mathrm{P}}$ are displayed in figure 1 . The exact transition amplitude for an inelastic process can be written in the form (see Taylor 1972, ch 18)

$$
W_{f i}=\left\langle\psi_{f}^{-}\left|V_{\mathrm{P}}+V_{\mathrm{N}}\right| \phi_{i}\right\rangle
$$




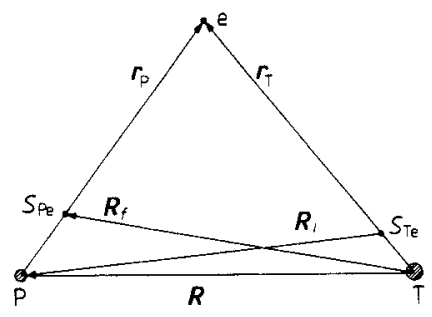

Figure 1. Coordinate system for a three-body problem consisting of projectile (P), target (T) and electron (e). The centres of mass of the projectile-electron and the target-electron systems are denoted by $S_{\mathrm{Pe}}$ and $S_{\mathrm{Te}}$, respectively.

where $\phi_{i}$ is an eigenstate of $T_{\mathrm{N}}+H_{\mathrm{T}}\left(H_{\mathrm{T}}=T_{\mathrm{e}}+V_{\mathrm{T}}\right), V_{\mathrm{P}}+V_{\mathrm{N}}$ is the scattering potential of the initial channel and $\psi_{f}^{-}$is the exact scattering solution to the three-body problem fulfilling the boundary condition that the electron is asymptotically in a bound projectile state while the projectile is in a quasi-elastic scattering state.

For asymmetric collision systems, $Z_{\mathrm{P}} \ll Z_{\mathrm{T}}$, one may expand $\psi_{f}^{-}$in terms of the weak field $V_{\mathrm{p}}$. Using the Faddeev equations (Faddeev 1961) for the representation of $\psi_{f}^{-}$the zero- and first-order approximations in $V_{\mathrm{P}}$ are given by

$$
\begin{aligned}
& \psi_{f}^{(0)-}=\left(1+G_{\mathrm{NT}}^{-} V_{\mathrm{T}}\right) \psi_{f} \\
& \psi_{f}^{(1)-}=\psi_{f}^{(0)-}+G_{\mathrm{NT}}^{-} V_{\mathrm{P}} \psi_{f}^{(0)-}
\end{aligned}
$$

where the Green's function is defined by $G_{\mathrm{NT}}^{-}=\left(E-H_{\mathrm{N}}-H_{\mathrm{T}}-\mathrm{i} \varepsilon\right)^{-1}$ and $E$ is the total energy of the collision system. The final-state wavefunction $\psi_{f}$ is a solution which includes the full internuclear Hamiltonian (eigenstate to $H_{\mathrm{N}}+H_{\mathrm{P}} ; H_{\mathrm{P}}=T_{\mathrm{e}}+V_{\mathrm{P}}$ ). As $\psi_{f}$ describes a state with an electron bound to the projectile at $t \rightarrow \infty$, the effect of projectile recoil is thus already contained in $\psi_{f}$ (see below).

When (2.3) is inserted into the transition amplitude (2.2) and all terms of equal order in $V_{\mathrm{P}}$ are collected, a formula analogous to the usual two-potential formula (see e.g. Taylor 1972) is obtained

$$
W_{f i} \simeq W_{f i}^{(0)}+W_{f i}^{(1)} \equiv\left\langle\psi_{f}^{(0)-}\left|V_{\mathrm{N}}\right| \phi_{i}\right\rangle+\left\langle\psi_{f}^{(0)-}\left|V_{\mathrm{P}}\right| \psi_{i}\right\rangle
$$

where $\psi_{i}$ is an exact solution to $H_{\mathrm{N}}+H_{\mathrm{T}}$. The first term $W_{f i}^{(0)}$ is of zero order in $V_{\mathrm{P}}$ and describes the electron capture through the internuclear potential. It is the quantum mechanical version of the target recoil amplitude (cf Feagin 1982 for the corresponding term in the case of ionisation). The first-order term $W_{f i}^{(1)}$ is the Coulomb capture amplitude. In the limit of $V_{\mathrm{N}}=0$, this term agrees with the plane-wave sPB approximation of Macek and co-workers $(1980,1982)$, while $W_{f i}^{(0)}$ vanishes. We point out that in formula (2.4) both the target field and the internuclear potential are retained to all orders. Thus it can be applied to asymmetric collision systems of medium or high projectile velocities.

For the evaluation of (2.4) it is crucial, as in any quantal treatment of three-body problems, to use the exact electronic and nuclear coordinates of the problem which for rearrangement collisions are different in the entrance and exit channel (cf figure 1):

$$
\begin{aligned}
& \boldsymbol{R}_{\mathrm{i}}=\boldsymbol{R}-\beta \boldsymbol{r}_{\mathrm{T}} \quad \beta=\frac{m}{m+M_{\mathrm{T}}} \\
& \boldsymbol{R}_{f}=\boldsymbol{R}+\alpha \boldsymbol{r}_{\mathrm{P}}=\boldsymbol{R}+\alpha\left(\boldsymbol{r}_{\mathrm{T}}-\boldsymbol{R}\right) \quad \alpha=\frac{m}{m+M_{\mathrm{P}}}
\end{aligned}
$$


where $m, M_{\mathrm{P}}$ and $M_{\mathrm{T}}$ are the mass of the electron, the projectile and the target nucleus, respectively. They give rise to important translation factors.

To find solutions for $\psi_{i}=\left(1+G_{\mathrm{NT}}^{+} V_{\mathrm{N}}\right) \phi_{i}$ we insert a complete set of eigenstates of $H_{\mathrm{T}}$ into the defining equation and use (2.5):

$$
\begin{aligned}
\psi_{i} & =\left(1+G_{\mathrm{NT}}^{+} V_{\mathrm{N}}\right) \sum_{n} \exp \left(\mathrm{i} \boldsymbol{K}_{i} \cdot \boldsymbol{R}\right) \varphi_{n}^{\mathrm{T}}\left(\boldsymbol{r}_{\mathrm{T}}\right)\left\langle\boldsymbol{\varphi}_{n}^{\mathrm{T}}\left|\exp \left(-\mathrm{i} \beta \boldsymbol{K}_{i} \cdot \boldsymbol{r}_{\mathrm{T}}\right)\right| \varphi_{i}^{\mathrm{T}}\right\rangle \\
& =\sum_{n} \hat{\chi}_{\boldsymbol{K}_{i}}^{+}\left(\omega_{n i}, \boldsymbol{R}\right) \varphi_{n}^{\mathrm{T}}\left(\boldsymbol{r}_{\mathrm{T}}\right)\left\langle\boldsymbol{\varphi}_{n}^{\mathrm{T}}\left|\exp \left(-\mathrm{i} \beta \boldsymbol{K}_{i} \cdot \boldsymbol{r}_{\mathrm{T}}\right)\right| \varphi_{i}^{\mathrm{T}}\right\rangle
\end{aligned}
$$

where we have used the defining equation for the internuclear off-shell wavefunction $\hat{\chi}_{K_{i}}$, whose energy, $\omega_{n i}=E-E_{n}^{\mathrm{T}}$, differs from its on-shell value by an amount $E_{n}^{\mathrm{T}}-E_{i}^{\mathrm{T}}$ determined by the strong potential. Here, $E_{i}^{\mathrm{T}}$ and $E_{n}^{\mathrm{T}}$ are the electronic energies in the initial and intermediate target states, respectively. However, if the collision is not more violent than that the matrix element $\left\langle\varphi_{n}^{\mathrm{T}}\left|\exp \left(-\mathrm{i} \beta \boldsymbol{K}_{i} \cdot \boldsymbol{r}_{\mathrm{T}}\right)\right| \varphi_{i}^{\mathrm{T}}\right\rangle$ can be treated in the dipole approximation, the contributions to (2.6) from $n \neq i$ will be proportional to $M_{\mathrm{P}} / M_{\mathrm{T}}$ and therefore of the order of $V_{\mathrm{P}}$. When $\psi_{i}$ is inserted into the transition amplitude (2.4), these contributions will be multiplied by another power of $V_{\mathrm{P}}$. It would thus be formally consistent to drop these terms in a lowest-order SPB approximation. There is, however, no technical advantage in dropping these contributions, provided we approximate the off-shell wavefunctions $\hat{\chi}_{\boldsymbol{K}_{i}}\left(\omega_{n i}, \boldsymbol{R}\right)$ by the corresponding on-shell wavefunction. The error this is likely to introduce will again be multiplied by $V_{\mathrm{P}} M_{\mathrm{P}} / M_{\mathrm{T}}$ and thus is of magnitude $\left(V_{\mathrm{P}}\right)^{2}$.

For the function $\psi_{f}$, an expression similar to (2.6) holds, with the target states $\varphi_{n}^{\mathrm{T}}$ replaced by projectile states. In this case, the nuclear scattering state is off-shell by an amount the scale of which is set by the weak potential. However, here and in the following we shall need the explicit assumption that the nuclear scattering amplitudes $f(K, \vartheta)$ vary slowly on the energy scale of the weak potential, i.e. we shall assume

$$
K \frac{\partial f(K, \vartheta)}{\partial E} \frac{1}{2} Z_{\mathrm{P}}^{2} \ll 1 .
$$

Thus, nuclear off-shell effects caused by the weak potential will consistently be neglected in the following. This is possible because, as will be discussed in the next section, we shall really be interested in the nuclear wavefunctions in their asymptotic region where, by definition, the normalisation of the off-shell states is the same as for on-shell states even in the presence of the internuclear Coulomb potential. With these remarks in mind, one can approximate the internuclear states in (2.6) - and its analogue for $\psi_{f}$-by on-shell wavefunctions (eigenstates of $H_{\mathrm{N}}$, defined with respect to the heavy-particle reduced mass $\mu$ ). Using the completeness relation one then finds

$$
\begin{aligned}
& \psi_{i}=\left(1+G_{\mathrm{NT}}^{+} V_{\mathrm{N}}\right) \phi_{i}=\varphi_{i}^{\mathrm{T}}\left(\boldsymbol{r}_{\mathrm{T}}\right) \chi_{\boldsymbol{K}_{i}}^{(+)}(\boldsymbol{R}) \exp \left(-\mathrm{i} \beta \boldsymbol{K}_{i} \cdot \boldsymbol{r}_{\mathrm{T}}\right) \\
& \psi_{f}=\left(1+G_{\mathrm{NP}}^{-} V_{\mathrm{N}}\right) \phi_{f}=\varphi_{f}^{\mathrm{P}}\left(\boldsymbol{r}_{\mathrm{P}}\right) \chi_{\boldsymbol{K}_{f}}^{(-)}(\boldsymbol{R}) \exp \left(\mathrm{i} \alpha \boldsymbol{K}_{f} \cdot \boldsymbol{r}_{\mathrm{P}}\right)
\end{aligned}
$$

Here $G_{\mathrm{NP}}^{-}=\left(E-H_{\mathrm{N}}-H_{\mathrm{P}}-\mathrm{i} \varepsilon\right)^{-1}, \varphi_{i}^{\mathrm{T}}$ and $\varphi_{f}^{\mathrm{P}}$ are the electronic initial and final bound states, respectively, and $\chi_{\boldsymbol{K}_{i}}$ and $\chi_{\boldsymbol{K}_{f}}$ are internuclear states of momentum $\boldsymbol{K}_{i}$ and $\boldsymbol{K}_{f}$. A derivation of the result $(2.8)$ with the use of on-shell internuclear states has recently been given by Végh (1983).

The presence of the translation factors $\exp \left(\mathrm{i} \alpha \boldsymbol{K}_{f} \cdot \boldsymbol{r}_{\mathrm{P}}\right)$ and $\exp \left(-\mathrm{i} \beta \boldsymbol{K}_{i} \cdot \boldsymbol{r}_{\mathrm{T}}\right)$ in (2.8) is related to the recoil which acts on the electron. The first factor is responsible for the projectile recoil effect, i.e. the fact that an electron which is bound to the projectile 
prior to the nuclear scattering, may have a large probability of changing its state when the projectile is scattered. This projectile recoil is proportional to the target mass and therefore a nonperturbative effect as $M_{\mathrm{T}} \gg M_{\mathrm{P}}$.

With the help of $(2.8)$ the function $\psi_{f}^{(0)-}$ can be evaluated by inserting a complete set of eigenfunctions to $H_{\mathrm{N}}+T_{\mathrm{e}}$ into (2.3)

$\psi_{f}^{(0)-}=\int \mathrm{d} \boldsymbol{K} \mathrm{d} \boldsymbol{q}\left(1+G_{\mathrm{NT}}^{-} V_{\mathrm{T}}\right)\left|\chi_{\boldsymbol{K}}^{(-)} \exp \left(-\mathrm{i} \beta \boldsymbol{K} \cdot \boldsymbol{r}_{\mathrm{T}}\right) \boldsymbol{q}\right\rangle\left\langle\chi_{\boldsymbol{K}}^{(-)} \exp \left(-\mathrm{i} \beta \boldsymbol{K} \cdot \boldsymbol{r}_{\mathrm{T}}\right) \boldsymbol{q} \mid \psi_{f}\right\rangle$

where the intermediate free electronic state $|\boldsymbol{q}\rangle$ is chosen to be in the target frame of reference. As demonstrated in appendix 1 , the application of $1+G_{\mathrm{NT}}^{-} V_{\mathrm{T}}$ leads to an electronic off-shell state $\psi_{q}(\omega)$, defined by

$$
\begin{aligned}
& \left(\omega-H_{\mathrm{T}}-\mathrm{i} \varepsilon\right) \psi_{\boldsymbol{q}}(\omega)=\left(\omega-q^{2} / 2-\mathrm{i} \varepsilon\right)|\boldsymbol{q}\rangle \\
& \omega=E-K^{2} / 2 \mu_{i} \quad \mu_{i}=\frac{M_{\mathrm{P}}\left(M_{\mathrm{T}}+m\right)}{M_{\mathrm{P}}+M_{\mathrm{T}}+m} \quad \varepsilon \rightarrow 0^{+} .
\end{aligned}
$$

Insertion into (2.4) then gives the result

$$
\begin{array}{rl}
W_{f i}^{(0)}=\int \mathrm{d} \boldsymbol{K} & \mathrm{d} \boldsymbol{q}\left\langle\chi_{\boldsymbol{K}_{f}^{(-)}}^{(-)} \varphi_{f}^{\mathrm{P}} \exp \left(\mathrm{i} \alpha \boldsymbol{K}_{f} \cdot \boldsymbol{r}_{\mathrm{P}}\right) \mid \chi_{\boldsymbol{K}}^{(-)} \exp \left(-\mathrm{i} \beta \boldsymbol{K} \cdot \boldsymbol{r}_{\mathrm{T}}\right) \boldsymbol{q}\right\rangle \\
& \times\left\langle\chi_{\boldsymbol{K}}^{(-)} \exp \left(-\mathrm{i} \beta \boldsymbol{K} \cdot \boldsymbol{r}_{\mathrm{T}}\right) \psi_{\boldsymbol{q}}(\omega)\left|V_{\mathrm{N}}\right| \varphi_{i}^{\mathrm{T}} \exp \left(\mathrm{i} \boldsymbol{K}_{i} \cdot \boldsymbol{R}\right) \exp \left(-\mathrm{i} \beta \boldsymbol{K}_{i} \cdot \boldsymbol{r}_{\mathrm{T}}\right)\right\rangle \\
W_{f \mathfrak{i}}^{(1)}=\int \mathrm{d} \boldsymbol{K} \mathrm{d} \boldsymbol{q}\left\langle\chi_{\boldsymbol{K}_{f}^{(-)}}^{(-)} \varphi_{f}^{\mathrm{P}} \exp \left(\mathrm{i} \alpha \boldsymbol{K}_{f} \cdot \boldsymbol{r}_{\mathrm{P}}\right) \mid \chi_{\boldsymbol{K}}^{(-)} \exp \left(-\mathrm{i} \beta \boldsymbol{K} \cdot \boldsymbol{r}_{\mathrm{T}}\right) \boldsymbol{q}\right\rangle \\
\\
\times\left\langle\chi_{\boldsymbol{K}}^{(-)} \exp \left(-\mathrm{i} \beta \boldsymbol{K} \cdot \boldsymbol{r}_{\mathrm{T}}\right) \psi_{\boldsymbol{q}}(\omega)\left|\boldsymbol{V}_{\mathrm{P}}\right| \varphi_{i}^{\mathrm{T}} \chi_{\boldsymbol{K}_{i}}^{(+)} \exp \left(-\mathrm{i} \beta \boldsymbol{K}_{\boldsymbol{i}} \cdot \boldsymbol{r}_{\mathrm{T}}\right)\right\rangle
\end{array}
$$

These formulae are the starting point for further evaluations. Both amplitudes $W_{f i}^{(0)}$ and $W_{f i}^{(1)}$ are expressed as a product of an excitation matrix element (resulting from the action of $V_{\mathrm{N}}$ and $V_{\mathrm{P}}$, respectively) and an overlap term, which describes the electron capture after excitation.

\section{Evaluation of the transfer amplitude}

An important simplification in the quantum mechanical treatment of atomic processes arises from the different length scales of the nuclear and the atomic wavefunctions. In general, the electronic potentials and functions vary slowly over an internuclear distance $R \leqslant R_{\mathrm{N}}$ where the nuclear wavefunctions deviate strongly from their asymptotic form. Thus it is convenient to decompose the nuclear states according to

$$
\begin{aligned}
& \chi_{\boldsymbol{K}}^{( \pm)}(\boldsymbol{R})=\chi_{\boldsymbol{K}}^{\mathrm{in}( \pm)}(\boldsymbol{R})+\chi_{\boldsymbol{K}}^{\mathrm{as}( \pm)}(\boldsymbol{R}) \\
& \chi_{\boldsymbol{K}}^{\mathrm{as}( \pm)}(\boldsymbol{R})=\exp (\mathrm{i} \boldsymbol{K} \cdot \boldsymbol{R})+f^{( \pm)}\left(\boldsymbol{K}, \vartheta_{\boldsymbol{K}, \boldsymbol{R}}\right) \frac{\exp ( \pm \mathrm{i} K R)}{R}
\end{aligned}
$$

where the asymptotic states $\chi_{K}^{\text {as }}$ have been expressed in terms of the scattering amplitude $f$ which depends on $\boldsymbol{K}$ and the angle $\vartheta_{K, \boldsymbol{R}}$ between $\boldsymbol{K}$ and $\boldsymbol{R}$. The logarithmic phase from the Coulomb part of $V_{\mathrm{N}}$ can be shown to be relatively unimportant (Rosenberg 1983) and has been neglected in the scattered wave. 
In the following we shall need matrix elements of operators $\hat{A}(\boldsymbol{r}, \boldsymbol{R})$ peaked at $\boldsymbol{r}=\boldsymbol{R}$. These matrix elements will get their main contribution from regions where $\chi$ may be replaced by $\chi^{\text {as }}$. However, for a resonance $\chi^{\text {in }}$ will also be important, but since the electronic wavefunctions are slowly varying in the region where $\chi^{\text {in }} \neq 0$, we can replace $\hat{A}(\boldsymbol{r}, \boldsymbol{R})$ by $\hat{A}(\boldsymbol{r}, 0)$ in all terms involving $\chi^{\text {in }}$. Thus, using the orthogonality of the nuclear states for different energies and the definition of the $S$ matrix (Taylor 1972), all matrix elements can be expressed by the asymptotic nuclear functions alone:

$$
\begin{array}{r}
\left\langle\chi_{\boldsymbol{K}^{\prime}}^{(-)}|\hat{A}(\boldsymbol{r}, \boldsymbol{R})| \chi_{\boldsymbol{K}}^{(+)}\right\rangle \simeq\left\langle\chi_{\boldsymbol{K}^{\prime}}^{\mathrm{as}(-)}|\hat{A}(\boldsymbol{r}, \boldsymbol{R})-\hat{A}(\boldsymbol{r}, 0)| \chi_{\boldsymbol{K}}^{\mathrm{as}(+)}\right\rangle+\hat{A}(\boldsymbol{r}, 0) S\left(\boldsymbol{K}, \boldsymbol{K}^{\prime}\right) \\
S\left(\boldsymbol{K}, \boldsymbol{K}^{\prime}\right)=\delta\left(\boldsymbol{K}-\boldsymbol{K}^{\prime}\right)+\frac{\mathrm{i}}{2 \pi \mu_{i}} \delta\left(E_{\boldsymbol{K}}-E_{\boldsymbol{K}^{\prime}}\right) f^{(+)}\left(\boldsymbol{K}, \boldsymbol{\vartheta}_{\boldsymbol{K}, \boldsymbol{K}^{\prime}}\right) .
\end{array}
$$

Here, $\boldsymbol{S}\left(\boldsymbol{K}, \boldsymbol{K}^{\prime}\right)$ is the nuclear scattering matrix and $E_{K}=K^{2} / 2 \mu_{i}$ the energy of the state $\chi_{K}$. If, instead of (3.2), the matrix element is taken between two ingoing (or two outgoing) states, $S\left(\boldsymbol{K}, \boldsymbol{K}^{\prime}\right)$ has to be replaced by $\delta\left(\boldsymbol{K}-\boldsymbol{K}^{\prime}\right)$. With the help of (3.2), the target recoil amplitude $W_{f i}^{(0)}$ and the Coulomb capture amplitude $W_{f i}^{(1)}$ can be readily evaluated.

\subsection{Target recoil amplitude}

Let us first consider the excitation matrix element in the definition (2.11) of $W_{f i}^{(0)}$. The electronic part, $\left\langle\psi_{\boldsymbol{q}}(\omega)\left|\exp \left(\mathrm{i} \beta\left(\boldsymbol{K}-\boldsymbol{K}_{i}\right) \boldsymbol{r}_{\mathrm{T}}\right)\right| \varphi_{i}^{\mathrm{T}}\right\rangle$, may be approximated by expanding the transition operator up to first order, because $\beta \simeq m / M_{\mathrm{T}}$ is a small quantity (dipole approximation). As the off-shell state $\psi_{q}(\omega)$ is not orthogonal to the initial state $\varphi_{i}^{\mathrm{T}}$, the zero-order term in the expansion does not vanish originally. However, with the usual choice of $\psi_{q}(\omega)$ as a renormalised Coulomb wave (Macek and Shakeshaft 1980)

$$
\psi_{q}(\omega) \simeq \exp (-\pi \eta / 2) \Gamma(1-\mathrm{i} \eta)\left(2 q^{2}\right)^{-\mathrm{i} \eta}\left(\omega-q^{2} / 2-\mathrm{i} \varepsilon\right)^{\mathrm{i} \eta} \varphi_{q}^{\mathrm{T}} \quad \eta=Z_{\mathrm{T}} / q
$$

only the first-order expansion term survives. It should be noted that the approximation (3.3) is only reasonable at short distances. Asymptotically, $\psi_{q}(\omega)$ and $\varphi_{q}^{\mathrm{T}}$ have the same normalisation. In the present case the momentum transfer $q$ will typically be of the order of $\boldsymbol{v}_{f}$ (the average electron velocity when it moves with the projectile), which will cut off the contributions to the matrix elements at distances larger than $q^{-1} \simeq v_{f}^{-1}$. Thus, this approximation is reasonable for intermediate and fast collisions $\left(v_{f} \geqslant Z_{\mathrm{T}}\right)$, which is the region where nuclear resonances are likely to be found.

The nuclear part of the excitation matrix element $\left\langle\chi_{\boldsymbol{K}}^{(-)}\left|V_{\mathrm{N}}\right| \exp \left(\mathrm{i} \boldsymbol{K}_{i} \cdot \boldsymbol{R}\right)\right\rangle$ can be expressed by the scattering amplitude. As shown in appendix 2, we thus obtain

$$
\begin{aligned}
& \left\langle\chi_{\boldsymbol{K}}^{(-)} \exp \left(-\mathrm{i} \beta \boldsymbol{K} \cdot \boldsymbol{r}_{\mathrm{T}}\right) \psi_{\boldsymbol{q}}(\omega)\left|V_{\mathrm{N}}\right| \varphi_{i}^{\mathrm{T}} \exp \left(\mathrm{i} \boldsymbol{K}_{i} \cdot \boldsymbol{R}\right) \exp \left(-\mathrm{i} \beta \boldsymbol{K}_{i} \cdot \boldsymbol{r}_{\mathrm{T}}\right)\right\rangle \\
& \simeq \frac{\mathrm{i} \beta}{4 \pi^{2} \mu_{i}}\left\langle\psi_{\boldsymbol{q}}(\omega)\left|\boldsymbol{r}_{\mathrm{T}}\right| \varphi_{i}^{\mathrm{T}}\right\rangle\left(\boldsymbol{K}_{i} f^{*(-)}\left(\boldsymbol{K}, \vartheta_{\boldsymbol{K}_{i},-\boldsymbol{K}}\right)-\boldsymbol{K} f^{(+)}\left(\boldsymbol{K}_{i}, \vartheta_{\boldsymbol{K}, \boldsymbol{K}_{i}}\right)\right) .
\end{aligned}
$$

The evaluation of the overlap term which enters into both $W_{f i}^{(0)}$ and $W_{f i}^{(1)}$ in $(2.11)$ is more involved. The electronic part simply yields the Fourier transform $\varphi_{f}^{* \mathrm{P}}(\boldsymbol{\kappa})$ of the final bound state

$\frac{1}{(2 \pi)^{3 / 2}} \int \mathrm{d} \boldsymbol{r}_{\mathrm{T}} \varphi_{f}^{* \mathrm{P}}\left(\boldsymbol{r}_{\mathrm{T}}-\boldsymbol{R}\right) \exp \left(\mathrm{i} \boldsymbol{\kappa} \cdot \boldsymbol{r}_{\mathrm{T}}\right)=\varphi_{f}^{* \mathrm{P}}(\boldsymbol{\kappa}) \exp (\mathrm{i} \boldsymbol{\kappa} \cdot \boldsymbol{R}) \quad \boldsymbol{\kappa}=\boldsymbol{q}-\alpha \boldsymbol{K}_{f}-\beta \boldsymbol{K}$. 
For the integration over $\boldsymbol{R}$, the relation (3.2) has to be used, with the electronic operator $\hat{A}(\boldsymbol{R})$ - the electronic coordinate $\boldsymbol{r}$ is already integrated out-given by $\exp [\mathrm{i}(\boldsymbol{q}-\boldsymbol{\beta} \boldsymbol{K}) \boldsymbol{R}]$. When evaluating the sticking term, which arises from the terms involving $\hat{A}(r, 0)$ in (3.2), a slight complication arises because $\exp (\mathrm{i} \boldsymbol{\kappa} \cdot \boldsymbol{R})$ from equation (3.5) does not satisfy the criterion of being peaked at $\boldsymbol{r} \simeq \boldsymbol{R}$, necessary to derive (3.2). However, we observe that from the conservation of the total energy $E$,

$$
E=K_{i}^{2} / 2 \mu_{i}+E_{i}^{\mathrm{T}}=K_{f}^{2} / 2 \mu_{f}+E_{f}^{\mathrm{P}} \quad \mu_{f}=\frac{M_{\mathrm{T}}\left(M_{\mathrm{P}}+m\right)}{M_{\mathrm{P}}+M_{\mathrm{T}}+m}
$$

where $E_{i}^{\mathrm{T}}$ and $E_{f}^{\mathrm{P}}$ are the energies of the initial and final electronic states, the kinetic energy $\left(v^{2} / 2\right)$ of the transferred electron is contained in the final energy $K_{f}^{2} / 2 \mu_{f}$ of the internuclear motion. Since the sticking term is a correction to the transfer amplitude, and $\exp (\mathrm{i} \boldsymbol{\kappa} \cdot \boldsymbol{R})$ incorporates a further correction to this term, we can neglect it provided we replace $\boldsymbol{K}_{f}$ by $\tilde{\boldsymbol{K}}_{f}=\boldsymbol{K}_{f}-\alpha \boldsymbol{K}_{f}$, in all terms associated with $\hat{\boldsymbol{A}}(\boldsymbol{r}, 0)$. This procedure is in the same spirit as the neglect of the off-shell effects in the nuclear wavefunction.

If the asymptotic wavefunctions are inserted from (3.1), the $\boldsymbol{R}$ integral can be carried out with the help of

$$
\begin{aligned}
\exp (\mathrm{i} \boldsymbol{K} \cdot \boldsymbol{R})= & \frac{2 \pi}{\mathrm{i} K R}\left[\exp (\mathrm{i} K R) \delta^{2}(\hat{\boldsymbol{R}}-\hat{\boldsymbol{K}})-\exp (-\mathrm{i} K R) \delta^{2}(\hat{\boldsymbol{R}}+\hat{\boldsymbol{K}})\right] \quad R \rightarrow \infty \\
& \int_{0}^{\infty} \mathrm{d} R \exp (\mathrm{i} Q R)=\pi \delta(Q)+\mathrm{i} P(1 / Q) .
\end{aligned}
$$

In the first relation which is only valid for large $R, \hat{\boldsymbol{K}}$ denotes the direction of $\boldsymbol{K}$. During the evaluation of the matrix elements, all terms involving sums of nuclear momenta in the exponent (like $\exp \left[\mathrm{i}\left(K+K^{\prime}\right) R\right]$ ) can be discarded because they describe the very unlikely process of the projectile backscattering on the electron. In the same spirit the (small) electronic momenta are only retained to first order

$$
|\boldsymbol{K}+\boldsymbol{\kappa}| \simeq K+\boldsymbol{\kappa} \cdot \hat{\boldsymbol{K}}
$$

if differences of the (large) nuclear momenta are involved, but neglected otherwise. This leads to the following expression for the overlap term $\left(\boldsymbol{\kappa}_{1}=\boldsymbol{q}-\boldsymbol{\beta} \boldsymbol{K}\right)$

$$
\begin{aligned}
W \equiv\left\langle\chi_{\boldsymbol{K}_{f}}^{(-)} \varphi_{f}^{\mathrm{P}}\right. & \exp \left[\mathrm{i} \alpha \boldsymbol{K}_{f}\left(\boldsymbol{r}_{\mathrm{T}}-\boldsymbol{R}\right)\right]\left|\chi_{\boldsymbol{K}}^{(-)} \exp \left(-\mathrm{i} \beta \boldsymbol{K} \cdot \boldsymbol{r}_{\mathrm{T}}\right) \boldsymbol{q}\right\rangle \\
= & \varphi_{f}^{* \mathrm{P}}(\boldsymbol{\kappa})\left[\delta\left(\boldsymbol{K}+\boldsymbol{\kappa}_{1}-\boldsymbol{K}_{f}\right)+\frac{\mathrm{i}}{4 \pi^{2} K} f^{*(-)}\left(\boldsymbol{K}_{f}, \vartheta_{\boldsymbol{K}_{f},-\boldsymbol{K}-\boldsymbol{\kappa}_{1}}\right)\right. \\
& \times\left(\pi \delta\left(K_{f}-K-\boldsymbol{\kappa}_{1} \cdot \hat{\boldsymbol{K}}\right)+\frac{\mathrm{i}}{K_{f}-K-\boldsymbol{\kappa}_{1} \cdot \hat{\boldsymbol{K}}}\right) \\
& -\frac{\mathrm{i}}{4 \pi^{2} K} f^{*(-)}\left(\tilde{\boldsymbol{K}}_{f}, \vartheta_{\boldsymbol{K}_{f},-\boldsymbol{K}}\right)\left(\pi \delta\left(\tilde{K}_{f}-K\right)+\frac{\mathrm{i}}{\tilde{K}_{f}-K}\right) \\
& -\frac{\mathrm{i}}{4 \pi^{2} K_{f}} f^{(-)}\left(\boldsymbol{K}, \vartheta_{\boldsymbol{K},-\boldsymbol{K}_{f}+\boldsymbol{\kappa}_{1}}\right)\left(\pi \delta\left(K_{f}-\boldsymbol{\kappa}_{1} \cdot \hat{\boldsymbol{K}}_{f}-K\right)+\frac{\mathrm{i}}{K_{f}-\boldsymbol{\kappa}_{1} \cdot \hat{\boldsymbol{K}}_{f}-K}\right) \\
& \left.+\frac{\mathrm{i}}{4 \pi^{2} \tilde{K}_{f}} f^{(-)}\left(\boldsymbol{K}, \vartheta_{\boldsymbol{K},-\boldsymbol{K}_{f}}\right)\left(\pi \delta\left(\tilde{K}_{f}-K\right)+\frac{\mathrm{i}}{\tilde{K}_{f}-K}\right)+\mathrm{O}\left(f^{2}\right)\right]
\end{aligned}
$$

The terms which contain a product of scattering amplitudes, i.e. which are of the order of $f^{2}$, correspond to a double nuclear scattering initiated by the electronic capture process. As the amplitude for large-angle deflection is small, these terms can be 
dropped. Similarly, when $W$ is inserted into the expression for $W_{f i}^{(0)}$, only the (threedimensional) $\delta$-function term is needed because the recoil matrix element (3.4) is linear in $f$.

From the time-reversal invariance of the scattering amplitude we have

$$
f^{*(-)}\left(\boldsymbol{K}, \vartheta_{\boldsymbol{K},-\boldsymbol{K}^{\prime}}\right)=f^{(+)}\left(\boldsymbol{K}, \vartheta_{\boldsymbol{K}, \boldsymbol{K}^{\prime}}\right)
$$

In order to compare with the semiclassical result, we express the nuclear momenta in terms of the initial and final projectile velocities

$$
\boldsymbol{K}_{i}=\mu_{i} \boldsymbol{v}_{i} \quad \boldsymbol{K}_{f}=\mu_{f} \boldsymbol{v}_{f}
$$

and discard small terms of the order of $m / M_{\mathrm{P}}$ or $m / M_{\mathrm{T}}$ in the resulting expression. Moreover, as the starting formula (2.4) is the first-order expansion term of the transition amplitude in terms of the weak projectile field, it is consistent to neglect also the terms proportional to $M_{\mathrm{P}} / M_{\mathrm{T}}$ because $M_{\mathrm{P}} / M_{\mathrm{T}} \leqslant Z_{\mathrm{P}} / Z_{\mathrm{T}}$. In particular, $\kappa$ then reduces to $\boldsymbol{q}-\boldsymbol{v}_{f}$. Thereby we have used that the target recoil term due to the proportionality to $\beta \boldsymbol{K}$ (cf equation (3.4)) is already of the order of $M_{\mathrm{P}} / M_{\mathrm{T}}$.

It is important, however, to neglect the above-mentioned terms only after any differences of nuclear momenta have been evaluated. With this in mind, the following relations can be found from the energy conservation (3.6):

$$
\begin{aligned}
& \mu_{f} \simeq \mu_{i}+1 \\
& v_{i}-v_{f} \approx \frac{1}{\mu_{i} v}\left(\Delta E+v^{2} / 2\right)
\end{aligned}
$$

where $\Delta E=E_{f}^{\mathrm{P}}-E_{i}^{\mathrm{T}}$ and $v_{i} \approx v_{f} \simeq v$ has been used when no differences are involved. The $\delta$ function from the first term in (3.9) determines the intermediate momentum $\boldsymbol{K}=\boldsymbol{K}_{f}-\boldsymbol{\kappa}_{1} \simeq \boldsymbol{K}_{\boldsymbol{f}}-\boldsymbol{q}$, so that the off-shell energy from (2.10) becomes $\omega \simeq$ $E_{i}^{\mathrm{T}}+\Delta E-v^{2} / 2+q v_{f}$. With this, the target recoil amplitude finally reduces to

$$
\begin{aligned}
W_{f i}^{(0)}=\int \mathrm{d} \boldsymbol{q} \varphi_{f}^{* \mathrm{P}} & \left(\boldsymbol{q}-\boldsymbol{v}_{f}\right) \frac{\mathrm{i}}{4 \pi^{2} M_{\mathrm{T}}}\left\langle\psi_{\boldsymbol{q}}(\omega)\left|\boldsymbol{r}_{\mathrm{T}}\right| \varphi_{i}^{\mathrm{T}}\right\rangle \\
& \times\left(\boldsymbol{v}_{i} f^{(+)}\left(\boldsymbol{K}_{f}-\boldsymbol{q}, \vartheta_{\boldsymbol{K}_{i}, \boldsymbol{K}_{f}-\boldsymbol{q}}\right)-\frac{1}{\mu_{i}}\left(\boldsymbol{K}_{f}-\boldsymbol{q}\right) f^{(+)}\left(\boldsymbol{K}_{\boldsymbol{i}}, \vartheta_{\boldsymbol{K}_{i}, \boldsymbol{K}_{f}-\boldsymbol{q}}\right)\right) .
\end{aligned}
$$

As in the case of ionisation, this formula may be interpreted in terms of the causal development of the collision process. In the last term, the nuclear scattering amplitude occurs with the incoming momentum $\boldsymbol{K}_{i}$, and thus the nuclear scattering takes place before the projectile has lost momentum to excite the electron. On the other hand, in the first term the nuclear scattering takes place with an intermediate momentum $\boldsymbol{K} \simeq \boldsymbol{K}_{f}-\boldsymbol{q}$ of the internuclear motion after excitation of the electron to the state $\psi_{\boldsymbol{q}}(\omega)$. However, since $\boldsymbol{K}_{f}-\boldsymbol{q} \neq \boldsymbol{K}_{f}$, the capture should not be regarded as having taken place yet. Thus this term corresponds to the semiclassical amplitude for excitation before, but capture after, the nuclear scattering (Amundsen and JakubassaAmundsen 1984a). Similar interpretations can also be given to the Coulomb capture amplitude, evaluated below. Note that the projectile momentum space wavefunction $\varphi_{f}^{\mathrm{P}}$ occurs with the same argument, $\boldsymbol{q}-\boldsymbol{v}_{f}$, for both amplitudes. This is the manifestation of the projectile recoil effect (Kocbach and Briggs 1984, Amundsen and JakubassaAmundsen 1984a).

Due to the presence of $\varphi_{f}^{\mathrm{P}}\left(\boldsymbol{q}-\boldsymbol{v}_{f}\right)$, the values of $\boldsymbol{q}$ will have a narrow distribution around $v_{f}$ with a width of the order of $Z_{\mathrm{p}}$. The application of Briggs' peaking which 
implies the replacement of $\boldsymbol{q}$ by $\boldsymbol{v}_{f}$ is thus consistent with our perturbation approach in the small parameter $Z_{\mathrm{p}}$, and also with the use of the on-shell limit (3.3) for $\psi_{\boldsymbol{q}}(\omega)$ and the condition (2.7). It has been applied to most of the SPB calculations reported so far (cf Macek and Alston 1982, Amundsen and Jakubassa-Amundsen 1984a). With this approximation, (3.13) reduces in the special case of capture from the target $\mathrm{K}$ shell into the projectile $1 \mathrm{~s}$ state (with energy $E_{f}^{\mathrm{P}}=-Z_{\mathrm{P}}^{2} / 2$ ) to the simple form

$$
\begin{aligned}
& W_{f i}^{(0)}=-\frac{2 \mathrm{i} \sqrt{\pi}}{M_{\mathrm{T}}} c_{0} \frac{v^{2}}{Z_{\mathrm{P}}}\left(1-\mathrm{i} \eta_{0}\right) \frac{\left[-\left(v+\mathrm{i} Z_{\mathrm{T}}\right)^{2}\right]^{-\mathrm{i} \eta_{0}}}{\left(Z_{\mathrm{T}}^{2}+v^{2}\right)^{2-\mathrm{i} \eta_{0}}} \\
& \times\left(\frac{1+\mathrm{i} \eta_{0}}{\left(v+\mathrm{i} Z_{\mathrm{T}}\right)^{2}}-\frac{2-\mathrm{i} \eta_{0}}{Z_{\mathrm{T}}^{2}+v^{2}}\right)\left(f^{(+)}\left(E_{i}, \vartheta\right)-\cos \vartheta f^{(+)}\left(E_{f}, \vartheta\right)\right) \\
& c_{0}=\frac{16 \mathrm{i}\left(2 v^{2}\right)^{\mathrm{i} \eta_{0}} \eta_{0}\left(Z_{\mathrm{P}} Z_{\mathrm{T}}\right)^{5 / 2}}{(2 \pi)^{2}\left[1-\exp \left(-2 \pi \eta_{0}\right)\right]}\left(\frac{2}{Z_{\mathrm{P}}^{2}}\right)^{\mathrm{i} \eta_{0}} \frac{\Gamma\left(\frac{1}{2}+\mathrm{i} \eta_{0}\right)}{\Gamma\left(2+\mathrm{i} \eta_{0}\right)} \quad \eta_{0}=Z_{\mathrm{T}} / v
\end{aligned}
$$

where the scattering angle $\vartheta$ is the angle between $\boldsymbol{v}_{i}$ and $\boldsymbol{v}_{f}$. The scattering energy $E_{f}$ is given by $E_{f} \equiv\left(\boldsymbol{K}_{f}-\boldsymbol{q}\right)^{2} / 2 \mu_{i} \simeq E_{i}-\left(\Delta E+v^{2} / 2\right.$ ) (with $\left.E_{i} \equiv K_{i}^{2} / 2 \mu_{i}\right)$. Thus the energies of the intermediate and the final internuclear states differ only by $\mathrm{O}\left(Z_{\mathrm{P}}^{2}\right)$.

\subsection{Coulomb capture amplitude}

The second contribution to the transfer amplitude, $W_{f_{i}}^{(1)}$, can be evaluated with the same techniques. If the Fourier representation is used for the projectile field $V_{\mathrm{P}}=$ $-Z_{\mathrm{P}} /\left|\boldsymbol{r}_{\mathrm{T}}-\boldsymbol{R}\right|$, the excitation matrix element turns out to be

$$
\begin{aligned}
M \equiv\left\langle\chi_{\boldsymbol{K}}^{(-)} \exp \right. & \left.\left(-\mathrm{i} \beta \boldsymbol{K} \cdot \boldsymbol{r}_{\mathrm{T}}\right) \psi_{q}(\omega)\left|V_{\mathrm{P}}\right| \varphi_{i}^{\mathrm{T}} \chi_{\boldsymbol{K}_{i}}^{(+)} \exp \left(-\mathrm{i} \beta \boldsymbol{K}_{i} \cdot \boldsymbol{r}_{\mathrm{T}}\right)\right\rangle \\
= & -\frac{Z_{\mathrm{P}}}{2 \pi^{2}} \int \frac{\mathrm{d} s}{s^{2}} M_{1}(\boldsymbol{q}, \boldsymbol{s})\left[\delta\left(\boldsymbol{K}_{i}-\boldsymbol{K}-\boldsymbol{s}\right)+\frac{\mathrm{i}}{4 \pi^{2} K^{\prime}} f^{(+)}\left(\boldsymbol{K}_{i}, \vartheta_{\boldsymbol{K}_{i}, \boldsymbol{K}+\boldsymbol{s}}\right)\right. \\
& \times\left(\pi \delta\left(K_{i}-K-s \cdot \hat{\boldsymbol{K}}\right)+\frac{\mathrm{i}}{K_{i}-K-\boldsymbol{s} \cdot \hat{\boldsymbol{K}}}\right) \\
& \left.+\frac{\mathrm{i}}{4 \pi^{2} K_{i}} f^{(+)}\left(\boldsymbol{K}, \vartheta_{\boldsymbol{K}, \boldsymbol{K}_{i}-\boldsymbol{s}}\right)\left(\pi \delta\left(K-K_{i}+\boldsymbol{s} \cdot \hat{\boldsymbol{K}}_{i}\right)+\frac{\mathrm{i}}{K-K_{i}+\boldsymbol{s} \cdot \hat{\boldsymbol{K}}_{i}}\right)\right] \\
& +M_{2}(\boldsymbol{q}) \frac{\mathrm{i} Z_{\mathrm{P}}}{4 \pi^{2}}\left[\frac{1}{K} f^{(+)}\left(\boldsymbol{K}_{i}, \vartheta_{\boldsymbol{K}_{i}, \boldsymbol{K}}\right)\left(\pi \delta\left(K_{i}-K\right)+\frac{\mathrm{i}}{K_{i}-K}\right)\right. \\
& +\frac{1}{K_{i}} f^{(+)}\left(\boldsymbol{K}, \vartheta_{\boldsymbol{K}_{i}, \boldsymbol{K}}\right)\left(\pi \delta\left(K_{i}-K\right)-\frac{\mathrm{i}}{K_{i}-K}\right) \\
& \left.-4 \pi \delta\left(K_{i}^{2}-K^{2}\right) f^{(+)}\left(\boldsymbol{K}_{i}, \vartheta_{\boldsymbol{K}_{i}, \boldsymbol{K}}\right)\right]
\end{aligned}
$$

with the abbreviations

$$
\begin{aligned}
M_{1}(\boldsymbol{q}, \boldsymbol{s})= & \left\langle\psi_{\boldsymbol{q}}(\omega) \exp \left(-\mathrm{i} \beta \boldsymbol{K} \cdot \boldsymbol{r}_{\mathrm{T}}\right)\left|\exp \left(\mathrm{i} \boldsymbol{s} \cdot \boldsymbol{r}_{\mathrm{T}}\right)\right| \varphi_{i}^{\mathrm{T}}\left(\boldsymbol{r}_{\mathrm{T}}\right) \exp \left(-\mathrm{i} \beta \boldsymbol{K}_{i} \cdot \boldsymbol{r}_{\mathrm{T}}\right)\right\rangle \\
& =\left\langle\psi_{\boldsymbol{q}}(\omega)\left|\exp \left(\mathrm{i} \boldsymbol{s} \cdot \boldsymbol{r}_{\mathrm{T}}\right)\right| \varphi_{i}^{\mathrm{T}}\right\rangle \\
M_{2}(\boldsymbol{q})= & \left\langle\psi_{\boldsymbol{q}}(\omega) \exp \left(-\mathrm{i} \beta \boldsymbol{K} \cdot \boldsymbol{r}_{\mathrm{T}}\right)\left|\frac{1}{r_{\mathrm{T}}}\right| \varphi_{i}^{\mathrm{T}}\left(\boldsymbol{r}_{\mathrm{T}}\right) \exp \left(-\mathrm{i} \beta \boldsymbol{K}_{i} \cdot \boldsymbol{r}_{\mathrm{T}}\right)\right\rangle \\
& =\left\langle\psi_{\boldsymbol{q}}(\omega)\left|\frac{1}{r_{\mathrm{T}}}\right| \varphi_{i}^{\mathrm{T}}\right\rangle .
\end{aligned}
$$


This has to be inserted, together with the expression (3.9) for the overlap term $W$, into the equation (2.11) for $W_{f i}^{(1)}$. The result can be arranged in terms which are of different order in the scattering amplitude $f$ : The zero-order term which arises from the product of the three-dimensional $\delta$ functions in $W$ and $M$ describes forward scattering and yields the main contribution to the total cross section, but can be neglected for large-angle scattering. Also, similarly as in the evaluation of the recoil amplitude, all terms quadratic in $f$ can be discarded. The two contributions to the linear terms in $f$ arise from either the $\delta$ function of $M$ (or $W$ ) together with the first-order terms in $f$ of $W$ (or $M$ ). This corresponds to the possibility of capture (or excitation) before and after the nuclear scattering. The resulting expression may be decomposed as

$$
W_{f i}^{(1)}=W_{f i}^{\mathrm{P}}+W_{f i}^{\mathrm{S}}+R_{f i}
$$

where $W_{f i}^{\mathrm{P}}$ collects all terms which survive when no resonance is present

$$
\begin{aligned}
W_{f i}^{\mathrm{P}}=-\frac{Z_{\mathrm{P}} \mathrm{i}}{8 \pi^{4} \mu_{i}} & \int \mathrm{d} \boldsymbol{q} \varphi_{f}^{* \mathrm{P}}\left(\boldsymbol{q}-\boldsymbol{v}_{f}\right) \int \frac{\mathrm{d} \boldsymbol{s}}{s^{2}} \hat{M}_{1}(\boldsymbol{q}, \boldsymbol{s})\left\{\left(E_{f}^{\mathrm{P}}-\frac{1}{2} v^{2}+\boldsymbol{q} \cdot \boldsymbol{v}_{f}-\frac{1}{2} q^{2}+\mathrm{i} \varepsilon\right)^{-\mathrm{i} \eta}\right. \\
& \times\left[f^{(+)}\left(\boldsymbol{K}_{i}, \vartheta_{\boldsymbol{K}_{i}, \boldsymbol{K}_{f}-\boldsymbol{q}+\boldsymbol{s}}\right)\right. \\
& \times\left(\pi \delta\left(\Delta E-\frac{1}{2} v^{2}+\boldsymbol{q} \cdot \boldsymbol{v}_{f}-\boldsymbol{s} \cdot \boldsymbol{v}_{f}\right)+\frac{\mathrm{i}}{\Delta E-\frac{1}{2} v^{2}-\boldsymbol{q} \cdot \boldsymbol{v}_{f}-\boldsymbol{s} \cdot \boldsymbol{v}_{f}}\right) \\
& \left.-f^{(+)}\left(\boldsymbol{K}_{f}-\boldsymbol{q}, \vartheta_{\boldsymbol{K}_{f}-\boldsymbol{q}, \boldsymbol{K}_{i}-\boldsymbol{s}}\right) \frac{\mathrm{i}}{\Delta E-\frac{1}{2} v^{2}+\boldsymbol{q} \cdot \boldsymbol{v}_{f}-\boldsymbol{s} \cdot \boldsymbol{v}_{i}}\right] \\
& +\left(E_{i}^{\mathrm{T}}+\boldsymbol{s} \cdot \boldsymbol{v}_{i}-\frac{1}{2} q^{2}+\mathrm{i} \varepsilon\right)^{-\mathrm{i} \eta}\left[f^{(+)}\left(\boldsymbol{K}_{f}, \vartheta_{\boldsymbol{K}_{f}, \boldsymbol{K}_{i}-\boldsymbol{s}+\boldsymbol{q}}\right)\right. \\
& \times\left(\pi \delta\left(\Delta E-\frac{1}{2} v^{2}-\boldsymbol{s} \cdot \boldsymbol{v}_{i}+\boldsymbol{q} \cdot \boldsymbol{v}_{i}\right)-\frac{\mathrm{i}}{\Delta E-\frac{1}{2} v^{2}-\boldsymbol{s} \cdot \boldsymbol{v}_{i}+\boldsymbol{q} \cdot \boldsymbol{v}_{i}}\right) \\
& \left.\left.+f^{(+)}\left(\boldsymbol{K}_{f}-\boldsymbol{v}_{f}, \boldsymbol{\vartheta}_{\boldsymbol{K}_{f}, \boldsymbol{K}_{i}-s}\right) \frac{\mathrm{i}}{\Delta E+\frac{1}{2} v^{2}-\boldsymbol{s} \cdot \boldsymbol{v}_{i}}\right]\right\} \\
& \hat{M}_{1}(\boldsymbol{q}, \boldsymbol{s})=\exp (-\pi \eta / 2) \Gamma(1+\mathrm{i} \eta)\left(2 q^{2}\right)^{\mathrm{i} \eta}\left\langle\boldsymbol{\varphi}_{q}^{\mathrm{T}}\left|\exp \left(\mathrm{i} \boldsymbol{s} \cdot \boldsymbol{r}_{\mathrm{T}}\right)\right| \boldsymbol{\varphi}_{i}^{\mathrm{T}}\right\rangle
\end{aligned}
$$

Here, the approximation (3.3) has been inserted for the off-shell state. As compared with the impulse approximation, where $\psi_{q}(\omega)$ in (3.15) is replaced by $\varphi_{q}^{\mathrm{T}}$, the structure of this expression is much more complex due to the off-shell phase factor which induces a coupling of the electronic and nuclear motion. Physically, this is related to the causal development of the transfer process.

All terms in (3.17) which are proportional to $f^{(+)}\left(\boldsymbol{K}_{f}-\boldsymbol{q}, \vartheta\right)$ or $f^{(+)}\left(\boldsymbol{K}_{f}-\boldsymbol{v}_{f}, \vartheta\right)$ correspond to excitation before, but capture after, the nuclear scattering, while the terms containing $f^{(+)}\left(\boldsymbol{K}_{i}, \vartheta\right)$ arise from both excitation and capture after the scattering. Such contributions were also present in the target recoil amplitude. However, the third kind of terms which are multiplied by $f^{(+)}\left(\boldsymbol{K}_{f}, \vartheta\right)$ are specific to $W_{f i}^{\mathrm{P}}$ and describe both excitation and capture before the nuclear interaction. It is these terms which have been influenced by the projectile recoil. 
The term $W_{f i}^{\mathrm{S}}$ is the sticking term which arises from the excitation matrix element in the inner region $R \leqslant R_{\mathrm{N}}$ :

$$
\begin{aligned}
W_{f i}^{\mathrm{S}}=\frac{Z_{\mathrm{p}} \mathrm{i}}{4 \pi^{2} \mu_{\mathrm{i}}} \int & \mathrm{d} \boldsymbol{q} \varphi_{f}^{* \mathrm{P}}\left(\boldsymbol{q}-\boldsymbol{v}_{f}\right) \exp (-\pi \eta / 2) \Gamma(1+\mathrm{i} \eta) \\
& \times\left(2 q^{2}\right)^{\mathrm{i} \eta}\left(E_{f}^{\mathrm{P}}-\frac{1}{2} v^{2}+\boldsymbol{q} \cdot \boldsymbol{v}_{f}-\frac{1}{2} q^{2}+\mathrm{i} \varepsilon\right)^{-\mathrm{i} \eta} \\
& \times\left\langle\boldsymbol{\varphi}_{\boldsymbol{q}}^{\mathrm{T}}\left|r_{\mathrm{T}}^{-1}\right| \varphi_{i}^{\mathrm{T}}\right\rangle\left(f^{(+)}\left(\boldsymbol{K}_{f}-\boldsymbol{q}, \vartheta_{\boldsymbol{K}_{i}, \boldsymbol{K}_{f}-\boldsymbol{q}}\right)-f^{(+)}\left(\boldsymbol{K}_{i}, \vartheta_{\boldsymbol{K}_{i}, \boldsymbol{K}_{f}-\boldsymbol{q}}\right)\right) \\
& \times\left(\pi \delta\left(\omega_{0}\right)-\frac{\mathrm{i}}{\omega_{0}}\right) \\
& \omega_{0}=\Delta E-v^{2} / 2+\boldsymbol{q} \cdot \boldsymbol{v}_{f} .
\end{aligned}
$$

It is proportional to the difference of the scattering amplitudes and is therefore only nonvanishing as long as $f$ varies appreciably with energy. This is also true for the remainder $R_{f i}$ which collects all terms not yet considered:

$$
\begin{aligned}
& R_{f i}=-\frac{Z_{\mathrm{P}} \mathrm{i}}{8 \pi^{4} \mu_{i}} \int \mathrm{d} \boldsymbol{q} \varphi_{f}^{* \mathrm{P}}\left(\boldsymbol{q}-\boldsymbol{v}_{f}\right) \int \frac{\mathrm{d} s}{s^{2}} \hat{M}_{1}(\boldsymbol{q}, \boldsymbol{s}) \\
& \times\left\{\left(E_{f}^{\mathbf{P}}-\frac{1}{2} v^{2}+\boldsymbol{q} \cdot \boldsymbol{v}_{f}-\frac{1}{2} q^{2}+\mathrm{i} \varepsilon\right)^{-\mathrm{i} \eta} f^{(+)}\left(\boldsymbol{K}_{f}-\boldsymbol{q}, \boldsymbol{\vartheta}_{\boldsymbol{K}_{f}-\boldsymbol{q}, \boldsymbol{K}_{i}-\mathbf{s}}\right)\right. \\
& \times \pi \delta\left(\Delta E-\frac{1}{2} v^{2}+q \cdot v_{f}-s \cdot v_{i}\right)+\left(E_{i}^{\mathrm{T}}+s \cdot v_{i}-\frac{1}{2} q^{2}+\mathrm{i} \varepsilon\right)^{-\mathrm{i} \eta} \\
& \times\left[-f^{(+)}\left(\boldsymbol{K}_{f}-\boldsymbol{v}_{f}, \boldsymbol{\vartheta}_{\boldsymbol{K}_{f}, \boldsymbol{K}_{i}-s}\right) \pi \delta\left(\Delta E+\frac{1}{2} v^{2}-\boldsymbol{s} \cdot \boldsymbol{v}_{i}\right)-f^{*(+)}\left(\boldsymbol{K}_{i}-\boldsymbol{s}, \boldsymbol{\vartheta}_{\boldsymbol{K}_{i}-\mathbf{s}, \boldsymbol{K}_{f}-\boldsymbol{q}}\right)\right. \\
& \times\left(\pi \delta\left(\Delta E-\frac{1}{2} v^{2}+\boldsymbol{q} \cdot \boldsymbol{v}_{f}-\boldsymbol{s} \cdot \boldsymbol{v}_{i}\right)-\frac{\mathrm{i}}{\Delta E-\frac{1}{2} v^{2}+q \cdot v_{f}-s \cdot v_{i}}\right) \\
& \left.\left.+f^{*(+)}\left(K_{i}-s, \vartheta_{K_{i}-s, K_{f}}\right)\left(\pi \delta\left(\Delta E+\frac{1}{2} v^{2}-s \cdot v_{i}\right)-\frac{\mathrm{i}}{\Delta E+\frac{1}{2} v^{2}-s \cdot v_{i}}\right)\right]\right\} \text {. }
\end{aligned}
$$

The difference between the energy denominators of the terms in (3.19) is given by $\boldsymbol{q}-\boldsymbol{v}_{f}$, i.e. is small of the order of $Z_{\mathrm{p}}$. Due to the mutual cancellations, the remainder is of higher order in $Z_{\mathrm{p}}$ than $W_{f i}^{\mathrm{P}}$ or $W_{f i}^{\mathrm{s}}$, and therefore very small. When Briggs' peaking, $q=v_{f}$, is applied, $R_{f i}$ vanishes identically.

With the peaking approximation, the sticking term can be evaluated in a way similar to that for the target recoil amplitude. As $\omega_{0}>0$, the only contribution comes from the principal value term in (3.18). For capture of a target $\mathrm{K}$ electron into the 1 s state of a hydrogen-like projectile, one finds

$$
W_{f i}^{\mathrm{s}}=\frac{c_{0}}{\mu_{i}} \frac{\mathrm{i} \sqrt{\pi}}{2 Z_{\mathrm{T}}} \frac{1}{\Delta E+\frac{1}{2} v^{2}} \frac{\left[-\left(v+\mathrm{i} Z_{\mathrm{T}}\right)^{2}\right]^{-\mathrm{i} \eta_{0}}}{\left(Z_{\mathrm{T}}^{2}+v^{2}\right)^{1-\mathrm{i} \eta_{0}}}\left(f^{(+)}\left(E_{i}, \vartheta\right)-f^{(+)}\left(E_{f}, \vartheta\right)\right)
$$

with the same definitions as given below (3.14).

\subsection{Comparison with the semiclassical formula}

In the absence of a resonance, or more precisely, if the nuclear scattering amplitude varies slowly on the energy scale of $E_{i}^{\mathrm{T}}$, so that $f^{(+)}\left(E_{i}, \vartheta\right) \simeq f^{(+)}\left(E_{f}, \vartheta\right)$, it can be taken 
outside all expressions as a common factor. In this case the sticking contribution $W_{f i}^{\mathrm{S}}$ vanishes and the target recoil becomes proportional to the corresponding semiclassical expression, with the proportionality constant $-f^{(+)}\left(E_{i}, \vartheta\right) /\left(4 \pi^{2} \mu_{i}\right)$. The reduction of $W_{f i}^{\mathrm{P}}$ to the semiclassical result involves an error of the order of $\left|\boldsymbol{q}-\boldsymbol{v}_{f}\right| \sim Z_{\mathrm{P}}$ which occurs in the last term of (3.17). Additional terms of this order have already been neglected, since the SPB theory is a first-order expansion in $Z_{\mathrm{p}}$. Incidentally, Briggs' peaking approximation gives a strict proportionality of $W_{f i}^{\mathrm{P}}$ with the semiclassical result (with the same constant as for $W_{f i}^{(0)}$ ). Thus the present quantal distorted-wave SPB reduces to the semiclassical theory in the zero impact parameter approximation, and the further evaluation of the transfer amplitude can be taken over from this case, as given by Amundsen and Jakubassa-Amundsen (1984a). In particular, the final form of $W_{f i}^{\mathrm{P}}$ is easily deduced from equations (3.7) and (3.11) of that work.

Although explicit formulae have only been given for the capture from the target $\mathrm{K}$ shell, the evaluation of the capture amplitudes from higher shells is straigh forward. When calculating the excitation matrix elements entering into (3.13) and (3.15) with the help of parametric differentiation of the 1 s ionisation matrix element (JakubassaAmundsen 1981), the same integrals emerge as given in the appendix of Amundsen and Jakubassa-Amundsen (1984a), although the resulting formulae for the capture amplitudes are somewhat more lengthy than for $\mathrm{K}$ capture.

\section{Charge transfer at isolated resonances}

In order to demonstrate the influence of a nuclear resonance on the capture probability we restrict ourselves to isolated resonances. Neglecting the background phase from the nonresonant scattering by the short-range part of $V_{N}$, as well as multichannel contributions to the resonance amplitude, the nuclear scattering amplitude for elastic scattering can be written in the form (Taylor 1972)

$$
\begin{aligned}
f^{(+)}\left(K^{\prime}, \vartheta^{\prime}\right)= & f_{\text {Coul }}-\frac{1}{2 K^{\prime}}(2 l+1) \exp \left(2 \mathrm{i} \sigma_{l}\right) P_{l}\left(\cos \vartheta^{\prime}\right) \frac{\Gamma_{\mathrm{P}}}{E^{\prime}-E_{\mathrm{R}}+\mathrm{i} \Gamma / 2} \\
& f_{\text {Coul }}=-\frac{\eta_{k}}{2 K^{\prime} \sin ^{2} \vartheta^{\prime} / 2} \exp \left(2 \mathrm{i} \sigma_{0}\right) \exp \left[-2 \mathrm{i} \eta_{k} \ln \left(\sin \vartheta^{\prime} / 2\right)\right]
\end{aligned}
$$

where $\eta_{k}=Z_{\mathrm{P}} Z_{\mathrm{T}} \mu_{i} / K^{\prime}, \sigma_{l}$ are the Coulomb phaseshifts of angular momentum $l$ and $P_{l}$ is a Legendre polynomial. Equation (4.1) is valid for $\mathrm{s}_{1 / 2}$ proton resonances as well as for impinging particles with spin zero. The resonance is described by a Breit-Wigner term characterised by the resonance energy $E_{R}$, the partial width $\Gamma_{P}$ for the decay of the compound system via its entrance channel and the total width $\Gamma$. For all resonances discussed below, $\Gamma_{\mathrm{p}}$ is equal to $\Gamma$. Further, $E^{\prime}=K^{\prime 2} / 2 \mu$.

When inserting (4.1) into the capture amplitude from the previous section, we make use of the fact that the Coulomb amplitude $f_{\text {Coul }}$ is a slowly varying function of $K^{\prime}$ and $\vartheta^{\prime}$ in the resonance region, and replace $K^{\prime}$ by the initial momentum $K_{i}$ and insert the scattering angle for $\vartheta^{\prime}$. For the simplest case of an $s_{1 / 2}$ resonance, the resonant part of (4.1) is $\vartheta^{\prime}$ independent, such that $f\left(K^{\prime}, \vartheta^{\prime}\right)$ appears even in the potential term $W_{f i}^{\mathrm{P}}$ only as an ( $s$ independent) factor. This is no longer true if $l \neq 0$, and there is no principal problem in performing the integrations when an $s$-dependent intermediate scattering angle emerges. However, the dependence on scattering angle is not likely to cause a rapid variation of the scattering amplitude, and because the electronic 
momenta produce only a small shift of the nuclear momenta $\boldsymbol{K}_{i}$ and $\boldsymbol{K}_{f}$, it is a reasonable approximation to neglect the $s$ dependence of $\vartheta^{\prime}$ and replace it by the scattering angle $\vartheta$. In this case the treatment of resonances with higher angular momenta becomes very simple and consists only in multiplying the Breit-Wigner factor for $s$ waves by $(2 l+1) \exp \left[2 \mathrm{i}\left(\sigma_{l}-\sigma_{0}\right)\right] P_{l}(\cos \vartheta)$. It may occur that in a very small energy region, the Coulomb amplitude is nearly cancelled by the resonance term in (4.1) which would lead to spurious narrow structures in the transition probability. In this case, the background phase has to be included in (4.1).

\section{Numerical results and discussion}

The probability for electron capture at a given scattering angle $\vartheta$ is found from

$$
P(\vartheta)=N_{0}(2 \pi)^{4} \mu_{i}^{2}\left|W_{f i}^{(0)}+W_{f i}^{\mathrm{P}}+W_{f i}^{\mathrm{S}}\right|^{2} \frac{1}{\left|f^{(+)}\left(K_{i}, \vartheta\right)\right|^{2}}
$$

where $N_{0}$ is the number of electrons in the initial target state. Since this formula reduces to the semiclassical expression discussed elsewhere (Amundsen and JakubassaAmundsen $1984 \mathrm{a}, \mathrm{b})$ if $f(K, \vartheta)$ is a slowly varying function of $K$, we shall present numerical results for nuclear resonant scattering only. Using experimental binding energies and Slater-screened hydrogen-like wavefunctions we have evaluated the capture from the $\mathrm{K}$ shell of ${ }^{22} \mathrm{Ne},{ }^{28} \mathrm{Si}$ and ${ }^{58} \mathrm{Ni}$ and from the $\mathrm{L}$ shell of ${ }^{58} \mathrm{Ni}$ by protons near an s-wave resonance, and also the capture from the $\mathrm{K}$ and $\mathrm{L}$ shell of ${ }^{20} \mathrm{Ne},{ }^{16} \mathrm{O}$ and ${ }^{28} \mathrm{Si}$ by alpha particles at a $3^{-}$resonance. Only transitions into the projectile ground state have been considered as they are strongly dominating for $Z_{\mathrm{P}} \ll Z_{\mathrm{T}}$, and Briggs' peaking approximation has been applied for the calculation of $P(\vartheta)$.

For the isotope ${ }^{22} \mathrm{Ne}$ there exist three s-wave resonances in the compound nucleus $\left({ }^{23} \mathrm{Na}\right)$ in a narrow energy region around 1.3-1.5 MeV (Endt and van der Leun 1973, $\mathrm{p} 67$ ). In figure 2 the capture probabilities near the resonance at $E_{\mathrm{P}}=1.513 \mathrm{MeV}$ with width $\Gamma=2.45 \mathrm{keV}$ are shown as a function of projectile energy. For this case, the energy transfer to the electron, $\delta E=E_{f}^{\mathrm{P}}-E_{i}^{\mathrm{T}}+v^{2} / 2$, is $1.7 \mathrm{keV}$ which is nearly equal to $\Gamma$. Correspondingly, the resonance structure of $P(\vartheta)$ is clearly visible, being about a factor of five enhanced over the background at scattering angles around $90^{\circ}$. The signal is reduced at small scattering angles because the $\sin ^{-2} \vartheta / 2$ behaviour of the Coulomb amplitude suppresses the Breit-Wigner term. For the other resonances at $E_{\mathrm{P}}=1.35$ and $1.378 \mathrm{MeV}$ the enhancement at $90^{\circ}$ is about one order of magnitude $(\Gamma / \delta E \simeq 1)$ and a factor of three $(\Gamma / \delta E \simeq 3)$, respectively. It is seen that for backward scattering angles the variation of $P(\vartheta)$ across the resonance is so strong that an effect should be measurable in a target of natural isotopic composition $\left(\approx 10 \%{ }^{22} \mathrm{Ne}\right)$.

Figure 3 shows the calculated transfer probability of $\mathrm{K}$ electrons from ${ }^{28} \mathrm{Si}$ in collisions with protons near the $s_{1 / 2}$ resonance at $2.083 \mathrm{MeV}$ (width $\Gamma=15.6 \mathrm{keV}$; Endt and van der Leun 1967, p 164). In this case, $\Gamma / \delta E=5.3$ and the change of $P(\vartheta)$ at the resonance position amounts up to $40 \%$. This makes it clear that the condition $\Gamma \simeq \delta E$ need not be strictly fulfilled in order to see an interference structure, however, the signal is the sharper, the closer $\Gamma / \delta E$ lies to unity. The variation of $P(\vartheta)$ with the scattering angle $\vartheta$ at fixed $E_{\mathrm{P}}$ expresses the dependence of $P(\vartheta)$ an the relative phase between the resonant part of the scattering amplitude and the Coulomb background. 


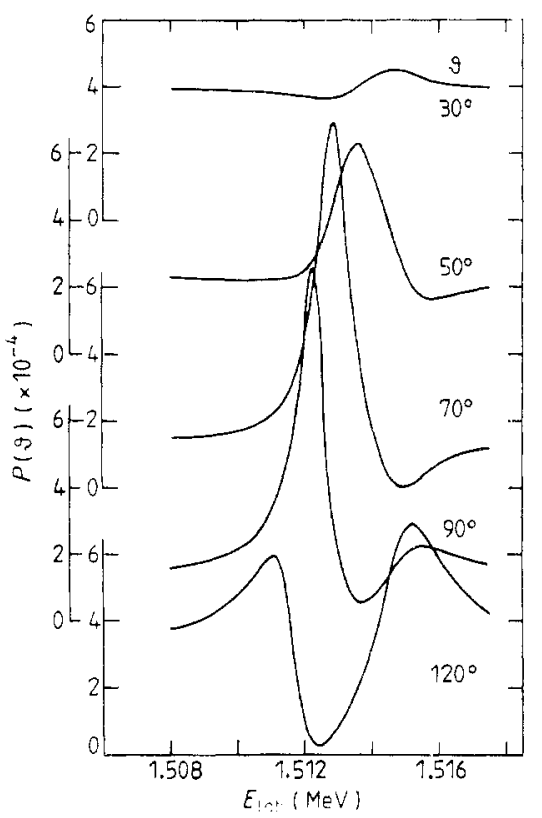

Figure 2. Capture probability of ${ }^{22} \mathrm{Ne} \mathrm{K}$-shell electrons by protons as a function of proton energy. The CM scattering angle $\vartheta$ is taken as the parameter. The uppermost scale on the left belongs to $\vartheta=30^{\circ}$, the next to $\vartheta=50^{\circ}$ and so on.

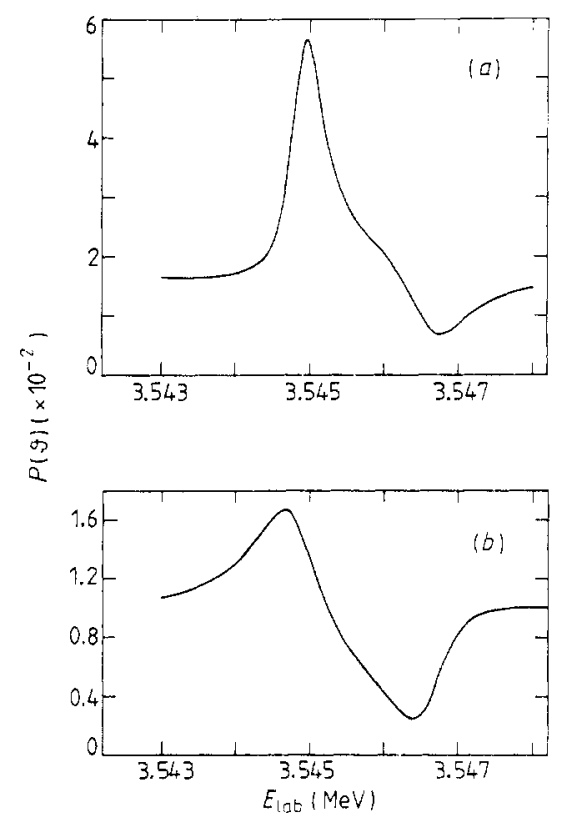

Figure 4. Capture probability of ${ }^{20} \mathrm{Ne} \mathrm{K}$-shell electrons by $\mathrm{He}^{2+}$ as a function of projectile energy. The $\mathrm{CM}$ scattering angle $\vartheta$ is: (a) $63.4^{\circ}$ and (b) $80^{\circ}$.

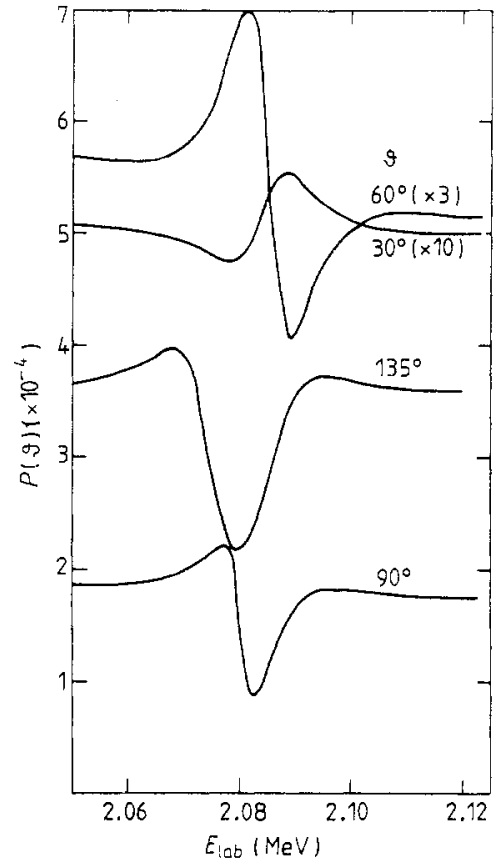

Figure 3. Capture probability of ${ }^{28} \mathrm{Si} \mathrm{K}$-shell electrons by protons as a function of proton energy. The CM scattering angle $\vartheta$ is taken as the parameter.

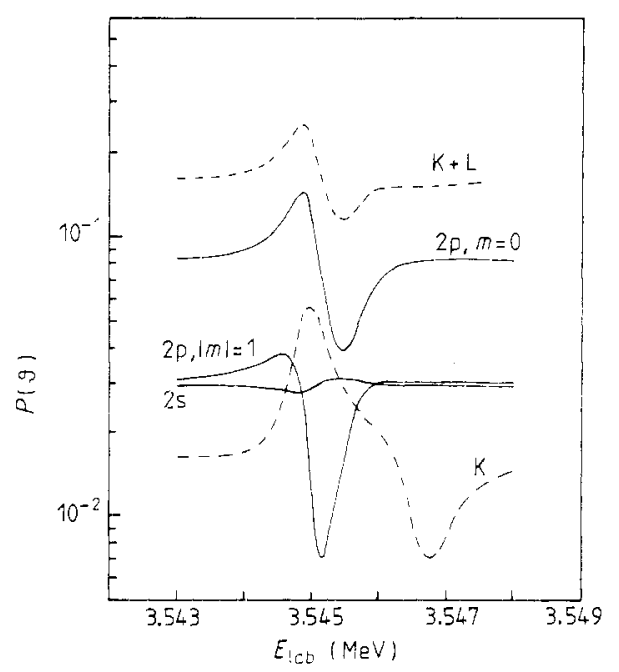

Figure 5. Capture probability in collisions of $\mathrm{He}^{2+}$ with ${ }^{20} \mathrm{Ne}$ as a function of projectile energy at $\vartheta=$ $63.4^{\circ}$. The full curves denote the capture probability from the Ne $L$ subshells, the broken curves are the $\mathrm{K}$-shell capture probability and the summed contributions from the $\mathrm{K}$ and $\mathrm{L}$ shells respectively. 
In figure 4 we have chosen a resonance in ${ }^{20} \mathrm{Ne}$ which is excited by alpha-particle impact $\left(E_{\alpha}=3.545 \mathrm{MeV}, \Gamma=1 \mathrm{keV}\right.$; Endt and van der Leun 1973, p 100). As it is a $3^{-}$resonance, the Breit-Wigner part of $f$ is weighted with the Legendre polynomial $P_{3}(\cos \vartheta)$, which has a zero at $\vartheta=90^{\circ}$. Thus the best choice for $\vartheta$ would be $63.4^{\circ}$ or $116.6^{\circ}$ where $P_{3}$ reaches its extrema. From figure 4 it is seen that for the smaller angle, the structure rises a factor of 3.5 above the background which is somewhat smaller than for the proton resonances in the case of a similar ratio of $\Gamma / \delta E=0.8$. However, a great advantage of the use of heavier projectiles lies in the fact that the capture cross section increases strongly with projectile charge $\left(\sim Z_{\mathrm{P}}^{5}\right.$; Jakubassa-Amundsen and Amundsen 1980). Thus, for the system ( $\alpha, N e \dot{)}$ the off-resonance capture probability from the target $\mathrm{K}$ shell lies in the per cent region compared with approximately $10^{-4}$ for proton impact of the same velocity.

The separation of $\mathrm{K}$ capture from other processes requires, apart from the observation of the charge state of the scattered projectile, either a high impact velocity (which exceeds the orbiting velocity of the target $\mathrm{K}$ electron) or a coincidence experiment with $\mathrm{K} \mathrm{x}$-rays or Auger electrons. In order to avoid such complications, we have investigated the question whether the resonance structure remains visible if electrons from higher shells are also included. In most cases, the impact energy is large enough so that only $\mathrm{K}$ - and L-shell electrons will contribute considerably to the capture probability. The obvious drawback of adding $\mathrm{K}$ - and L-shell capture is twofold: first, as the $L$ binding energy is smaller, the ratio $\Gamma / \delta E$ gets less favourable (in nearly all cases $\Gamma$ is already larger than the energy transfer for the $K$ shell), and second, the total capture probability is a sum over four partial probabilities ( $\mathrm{K}$ shell $+\mathrm{L}$ subshells), which will tend to wash out the resonance structure.

A study of L-shell capture from Ne by $\alpha$ impact shows that at $\vartheta=63.4^{\circ}$ the dominant contribution arises from the target $2 \mathrm{p}, m=0$ state (figure 5). The total $\mathrm{K}+\mathrm{L}$-shell capture probability $P(\vartheta) \simeq 0.15$ and shows a $60 \%$ variation across the resonance.

Figure 6 shows $P(\vartheta)$ for $\alpha$ colliding with ${ }^{16} \mathrm{O}$ near the resonance at $5.486 \mathrm{MeV}$ ( $\Gamma=4 \mathrm{keV}$; Ajzenberg-Selove 1978, p 165). At this rather high impact velocity, the capture from the oxygen $\mathrm{K}$ shell is dominating; however, as $\Gamma / \delta E_{\mathrm{K}}=3.2$, the variation of $P(\vartheta)$ is not so large as for the $\mathrm{Ne} \mathrm{K}$ shell. The use of hydrogen-like wavefunctions may be questionable for the $\mathrm{L}$ shell of these light systems, but improved wavefunctions will mainly affect the background value of $P(\vartheta)$ and not the resonance structure.

In figure 7 results are shown for $\mathrm{K}$ - and L-subshell capture in the case of the 'historical' resonance of $\mathrm{p} \rightarrow{ }^{58} \mathrm{Ni}$ at $E_{\mathrm{p}}=3.151 \mathrm{MeV}$ with width $\Gamma=5.6 \mathrm{keV}$ which was the first one across which ionisation probabilities have been measured (Blair et al 1978). The structure of the L-subshell capture probabilities is seen to be nearly as large as for $\mathrm{K}$-shell capture, because in this special case $\Gamma / \delta E_{\mathrm{L}}=2.2$ is roughly the inverse of $\Gamma / \delta E_{\mathrm{K}}=0.56$, and also the peak position is at about the same energy. Thus, even the summed $\mathrm{K}+\mathrm{L}$ capture probability shows a large structure and is one order of magnitude above the $\mathrm{K}$ capture probability. The suppression of the $2 \mathrm{p}, m=0$ subshell capture is due to the specific choice of $\vartheta=90^{\circ}$ where excitation perpendicular to the beam direction $(m=1)$ is enhanced. In order to investigate resonances with a larger width, heavier target systems should be chosen where the binding energy and thus $\delta E_{\mathrm{K}}$ is larger. For alpha projectiles one can probably not go much beyond ${ }^{28} \mathrm{Si}$ without risking that the compound system decays preferentially via other channels than (elastic) alpha emission. For this system we have calculated the L-shell capture probability across the $3^{-}$resonance at $E_{\alpha}=5.58 \mathrm{MeV}$ with width $10 \mathrm{keV}$ (Stautberg et al 1968). In this case, $\Gamma / \delta E_{\mathrm{L}}(=12.4)$ is much larger than for the $\mathrm{K}$ shell $\left(\Gamma / \delta E_{\mathrm{K}}=3.9\right)$. 


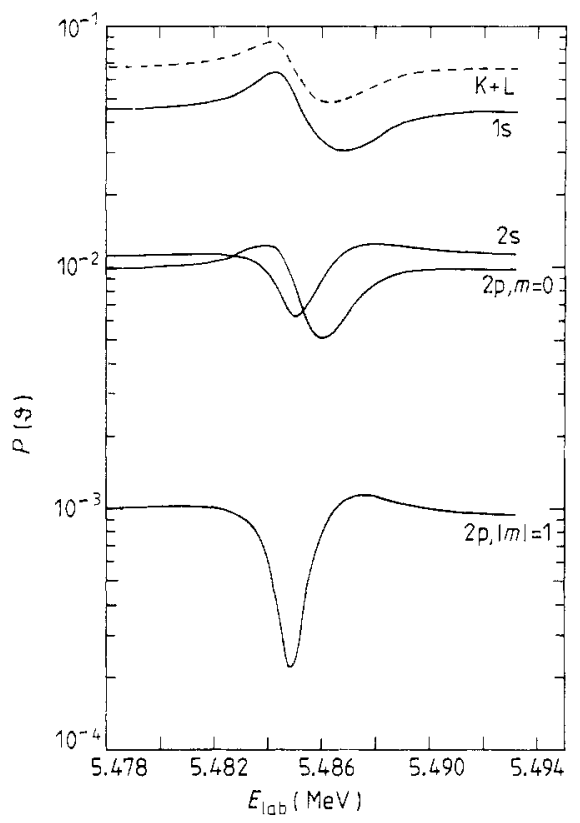

Figure 6. Capture probability in collisions of $\mathrm{He}^{2+}$ with ${ }^{16} \mathrm{O}$ as a function of projectile energy at $\vartheta=65^{\circ}$. Given are the $\mathrm{K}$ and $\mathrm{L}$ subshell capture probabilities (full curves) and their sum (broken curve).

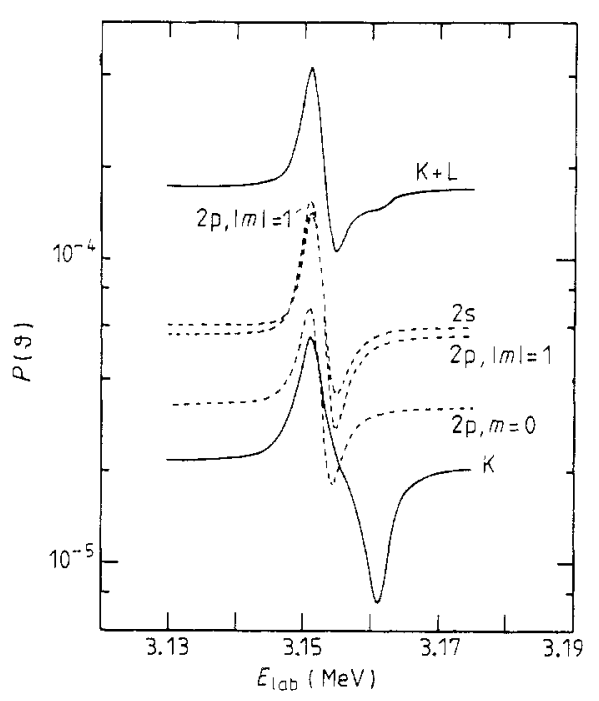

Figure 7. Capture probability in collisions of protons with ${ }^{58} \mathrm{Ni}$ as a function of proton energy at $\vartheta=90^{\circ}$. Shown is the capture probability from the Ni K-shell (full curve denoted by $\mathrm{K}$ ) and from the L subshells (broken curves). Also shown is their sum (full curve denoted by $\mathrm{K}+\mathrm{L}$ ).

Although the resonance effect is rather strong for the $\mathrm{K}$ shell, the variation in the total $\left(\mathrm{K}+\mathrm{L}\right.$ ) capture probability is only about $20 \%$ at $\vartheta=65^{\circ}$, and $P(\vartheta) \simeq 0.06$. At backward angles $\left(115^{\circ}-130^{\circ}\right)$ the signal is somewhat more pronounced.

From our chosen examples it follows that the condition $\Gamma \simeq \delta E$ is more stringent for the $\mathrm{L}$ shell than for the $\mathrm{K}$ shell in order to observe a resonant behaviour of $P(\vartheta)$. Note that for the $\mathrm{L}$ shell the presence of the term $v^{2} / 2$ in $\delta E$ makes the 'time-delay' effects in capture much larger for broad (on an atomic scale) resonances than is the case in ionisation.

\section{Conclusion}

We have developed a distorted-wave sPB theory in a fully quantum mechanical treatment, which allows the effect of resonant nuclear scattering on the capture of target electrons to be included. In calculating some test cases of proton and alpha impact on heavier targets we have shown that charge transfer is a promising tool to study nuclear resonance effects, because the interference structure is strongly visible on the smooth background even in cases where the energy transferred to the electron is not equal to the energy scale $\Gamma$ provided by the width of the resonance.

Once this method is established experimentally for known isolated resonances which provide a crucial test for the atomic theory for charge transfer-due to the sensitivity on the relative phases of the capture amplitudes in the ingoing and outgoing channel-more complicated resonances may be investigated in order to get also 
information on the nuclear scattering amplitudes. A generalisation of our theory to other resonances, such as overlapping isobaric analogue states, is, in principle, straightforward.

\section{Appendix 1}

We prove that the application of $\left(1+G_{\mathrm{NT}}^{-} V_{\mathrm{T}}\right)$ on an eigenfunction $\psi_{\boldsymbol{K}}$ to $H_{\mathrm{N}}+T_{\mathrm{e}}$ leads to an electronic off-shell function as defined by equation (2.10). We thereby use the on-shell approximation for $\psi_{\boldsymbol{K}}$, namely $\left|\psi_{\boldsymbol{K}}\right\rangle=\chi_{\boldsymbol{K}}^{(-)} \exp \left(-\mathrm{i} \boldsymbol{\beta} \boldsymbol{K} \cdot \boldsymbol{r}_{\mathrm{T}}\right)|\boldsymbol{q}\rangle$. Introducing an electronic state $\psi_{q}(\omega)$ by means of

$$
\left(1+G_{\mathrm{NT}}^{-} V_{\mathrm{T}}\right) \psi_{\boldsymbol{K}}=\chi_{\boldsymbol{K}}^{(-)} \exp \left(-\mathrm{i} \beta \boldsymbol{K} \cdot \boldsymbol{r}_{\mathrm{T}}\right) \psi_{\boldsymbol{q}}(\omega)
$$

and inserting a complete set of electronic plane waves $|\boldsymbol{p}\rangle$ before $\psi_{\boldsymbol{q}}(\omega)$ we obtain after multiplication with $\left(G_{\mathrm{NT}}^{-}\right)^{-1}$ from the left

$$
\begin{aligned}
\left(E-H_{\mathrm{N}}-\right. & \left.T_{\mathrm{e}}-\mathrm{i} \varepsilon\right) \psi_{\boldsymbol{K}} \\
= & \sum_{p}\left(E-H_{\mathrm{N}}-T_{\mathrm{e}}-\mathrm{i} \varepsilon\right) \chi_{\boldsymbol{K}}^{(-)} \exp \left(-\mathrm{i} \beta \boldsymbol{K} \cdot \boldsymbol{r}_{\mathrm{T}}\right)|\boldsymbol{p}\rangle\left\langle\boldsymbol{p} \mid \psi_{\boldsymbol{q}}(\omega)\right\rangle \\
& \quad-V_{\mathrm{T}} \chi_{\boldsymbol{K}}^{(-)} \exp \left(-\mathrm{i} \beta \boldsymbol{K} \cdot \boldsymbol{r}_{\mathrm{T}}\right) \psi_{\boldsymbol{q}}(\omega) .
\end{aligned}
$$

Using that $\psi_{\boldsymbol{K}}$ and $\chi_{K}^{(-)} \exp \left(-\mathrm{i} \beta \boldsymbol{K} \cdot \boldsymbol{r}_{\mathrm{T}}\right)|\boldsymbol{p}\rangle$ are eigenstates of $H_{\mathrm{N}}+T_{\mathrm{e}}$ with energy $K^{2} / 2 \mu_{i}+q^{2} / 2$ and $K^{2} / 2 \mu_{i}+p^{2} / 2$, respectively, all operators with respect to the nuclear coordinate $\boldsymbol{R}$ have disappeared. Thus the nuclear function $\chi_{\boldsymbol{K}}^{(-)}$may be dropped:

$$
\begin{aligned}
\left(E-K^{2} / 2 \mu_{i}-\right. & \left.q^{2} / 2-\mathrm{i} \varepsilon\right) \exp \left(-\mathrm{i} \beta \boldsymbol{K} \cdot \boldsymbol{r}_{\mathrm{T}}\right)|\boldsymbol{q}\rangle \\
= & \sum_{p}\left(E-K^{2} / 2 \mu_{i}-p^{2} / 2-\mathrm{i} \varepsilon\right) \exp \left(-\mathrm{i} \beta \boldsymbol{K} \cdot \boldsymbol{r}_{\mathrm{T}}\right)|\boldsymbol{p}\rangle\left\langle\boldsymbol{p} \mid \psi_{\boldsymbol{q}}(\omega)\right\rangle \\
& \quad-V_{\mathrm{T}} \exp \left(-\mathrm{i} \beta \boldsymbol{K} \cdot \boldsymbol{r}_{\mathrm{T}}\right) \psi_{\boldsymbol{q}}(\omega) .
\end{aligned}
$$

Upon multiplication by $\exp \left(+\mathrm{i} \beta \boldsymbol{K} \cdot \boldsymbol{r}_{\mathrm{T}}\right)$ from the left and subsequently using that $p^{2} / 2|\boldsymbol{p}\rangle=T_{\mathrm{e}}|\boldsymbol{p}\rangle$, we obtain finally after using closure:

$$
\left(E-K^{2} / 2 \mu_{i}-q^{2} / 2-\mathrm{i} \varepsilon\right)|\boldsymbol{q}\rangle=\left(E-K^{2} / 2 \mu_{i}-T_{\mathrm{e}}-V_{\mathrm{T}}-\mathrm{i} \varepsilon\right) \psi_{\boldsymbol{q}}(\omega)
$$

which makes clear that $\psi_{q}(\omega)$ is the desired off-shell function.

\section{Appendix 2}

We show that the excitation matrix element which originates from the target recoil can be written in the form (3.4).

In the dipole approximation, we have to evaluate

$$
I \equiv\left(\boldsymbol{K}-\boldsymbol{K}_{i}\right)\left\langle\chi_{\boldsymbol{K}}^{(-)}\left|V_{\mathrm{N}}\right| \exp \left(\mathrm{i} \boldsymbol{K}_{i} \cdot \boldsymbol{R}\right)\right\rangle .
$$

As $\chi_{K}$ is eigenstate to the nuclear Hamiltonian $H_{\mathrm{N}}=T_{\mathrm{N}}+V_{\mathrm{N}}, I$ can be written in a symmetrised form, using $\left|\chi_{\boldsymbol{K}}\right\rangle=\left(1+G_{\mathrm{N}} V_{\mathrm{N}}\right) \exp (\mathbf{i} \boldsymbol{K} \cdot \boldsymbol{R})$

$$
I=\boldsymbol{K}\left\langle\exp (\mathrm{i} \boldsymbol{K} \cdot \boldsymbol{R})\left|V_{\mathrm{N}}\right| \chi_{\boldsymbol{K}_{i}}^{(+)}\right\rangle-\boldsymbol{K}_{i}\left\langle\chi_{\boldsymbol{K}}^{(-)}\left|V_{N}\right| \exp \left(\mathrm{i} \boldsymbol{K}_{i} \cdot \boldsymbol{R}\right)\right\rangle
$$

where $\chi_{K_{i}}^{(+)}$is the ingoing scattering state of $H_{\mathrm{N}}$. For elastic scattering $\left(K=K_{i}\right)$ the nuclear matrix elements can be expressed in terms of the scattering amplitude 
$f^{+1}\left(\boldsymbol{K}_{i}, \vartheta_{\boldsymbol{K}, \boldsymbol{K}_{i}}\right)$. For almost elastic processes we thus have

$I=-\frac{1}{(2 \pi)^{2} \mu_{i}}\left(\boldsymbol{K} f^{(+)}\left(\boldsymbol{K}_{i}, \vartheta_{\boldsymbol{K}, \boldsymbol{K}_{i}}\right)-\boldsymbol{K}_{i} f^{*(-)}\left(\boldsymbol{K}, \vartheta_{\boldsymbol{K}_{\mathrm{i}},-\boldsymbol{K}}\right)\right)+\mathrm{O}\left(\frac{\left(\boldsymbol{K}_{i}-K\right)^{2}}{K_{i}^{2}}\right)$

from which one immediately gets (3.4).

\section{References}

Ajzenberg-Selove F 1978 Nucl. Phys. A 3001

Amundsen P A and Jakubassa-Amundsen D H 1984a J. Phys. B: At. Mol. Phys. 172671 1984b Phys. Rev. Lett. 53222

Anholt R, Chemin J F and Amundsen P A 1982 Phys. Lett. 118B 245

Blair J S and Anholt R 1982 Phys. Rev. A 25907

Blair J S, Dyer P, Snover K A and Trainor T A 1978 Phys. Rev. Lett. 411712

Chemin J F, Andriamonje S, Morenzoni E, Stoller Ch, Anholt R, Meyerhof W E, Baker O K and Molitoris J D 1983 Phys. Lett. 130B 246

Chemin J F, Anholt R, Stoller Ch, Meyerhof W E and Amundsen P A 1981 Phys. Rev. A 241218

Chemin J F, Meyerhof W E, Anholt R, Molitoris J D and Stoller Ch 1982 Phys. Rev. A 261239

Ciocchetti $G$ and Molinari A 1965 Nuovo Cim. B 4069

Endt P M and van der Leun C 1967 Nucl. Phys. A 1051

1973 Nucl. Phys. A 2141

Faddeev L D 1961 Sov. Phys.-JETP 121014

Feagin J M 1982 J. Phys. B: At. Mol. Phys. 153721

Feagin J M and Kocbach L 1981 J. Phys. B: At. Mol. Phys. 144349

Horsdal Pedersen E, Cocke C L, Rasmussen J L, Varghese S L and Waggoner W 1983 J. Phys. B: At. Mol. Phys. 161799

Horsdal Pedersen E, Loftager P and Rasmussen J L 1982a J. Phys. B: At. Mol. Phys. 152461

1982b J. Phys. B: At. Mol. Phys. 154423

Jakubassa-Amundsen D H 1981 J. Phys. B: At. Mol. Phys. 142647 1984 Z. Phys. A 316161

Jakubassa-Amundsen D H and Amundsen P A 1980 Z. Phys. A 297203 1981 J. Phys. B: At. Mol. Phys. 14 L705

Kocbach L and Briggs J S 1984 J. Phys. B: At. Mol. Phys. 173255

Macek J and Alston S 1982 Phys. Rev. A 26250

Macek J H and Shakeshaft R 1980 Phys. Rev. A 221441

Macek J and Taulbjerg K 1981 Phys. Rev. Lett. 46170

McVoy K W and Weidenmüller H A 1982 Phys. Rev. A 251462

Meyerhof W E, Astner G, Hofmann D, Groeneveld K O and Chemin J F 1982 Z. Phys. A 30993

Rosenberg L 1983 Phys. Rev. A 283238

Stautberg M M, Cosack M, Schulte R L, Leung M K, Weil J L and McEllistrem M T 1968 Bull. Am. Phys. Soc. 13674

Taylor J R 1972 Scattering Theory (New York: Wiley) ch 3b, $14 b$

Végh L 1983 J. Phys. B: At. Mol. Phys. 164175 